\title{
CHANGING THE TYPE OF KNOWLEDGE OF RESULTS AFFECTS THE LEARNING OF A LINE-DRAWING TASK
}

original paper

() Wroclaw University of Health and Sport Sciences

DOI: https://doi.org/10.5114/hm.2021.104187

\section{KEVIN M. FISHER, WESTON KARTES, MEGAN GREGORSKI}

Central Michigan University, Mount Pleasant, Michigan, USA

\begin{abstract}
Purpose. Augmented feedback is information that is inherently unavailable to a learner and must be provided by an outside source such as an expert or technical display. Such feedback may be divided into knowledge of performance, relating to movement quality, and knowledge of results (KR), relating to a movement outcome. KR has been examined with respect to variables such as timing, medium, and precision. In previous research involving a line-drawing task, individuals presented with higher levels of KR precision outperformed those presented with distracting feedback (nonsense syllables) or none. The present study sought to extend these findings by comparing the effects of KR types on learning a line-drawing task. Methods. On day 1, participants $(n=48)$ practised in 4 groups, receiving unique extrinsic feedback: control (no feedback), vague, precise, and visual feedback group. On day 2, learning was assessed via retention and transfer testing.

Results. For acquisition, a repeated-measures ANOVA revealed a main effect for block in absolute constant error and variable error $(p<0.01)$, indicating improved accuracy and consistency with practice. During testing, results showed a main effect such that accuracy and consistency during retention were better than transfer $(p<0.01)$. Follow-up pairwise comparisons indicated significantly better performance for the visual feedback group when compared with the control group ( $p=0.021)$. Conclusions. These results suggest that learning a simple task such as line-drawing may improve through verbal or visual feedback and that the latter medium may be an effective alternative to feedback that is presented verbally.
\end{abstract}

Key words: feedback, knowledge of results (KR), motor learning

\section{Introduction}

Sensory information is generated throughout movement, including periods before, during, and after the action [1]. Information regarding current or past aspects of a performance that may be used as a basis for improvement is termed feedback and may be subdivided into 2 main categories: intrinsic and extrinsic $[2,3]$. Intrinsic feedback (i.e., response-produced feedback) is the internal sensory information received from movement, while extrinsic feedback (i.e., augmented feedback) is information received from an outside source such as an expert or technical display. Although intrinsic feedback is inherently available to a learner, extrinsic feedback often comes from experts such as coaches or educators or a technical display that can analyse motor patterns or the movement results such as a video camera or a launch monitor in golf [4]. Feed- back is critical to skill learning because it provides valuable information to improve performance and acts as a tool for learning to continue [1]. Additionally, it can enhance performer motivation and reinforce desirable behaviours while reducing the occurrence of undesirable behaviours [5].

Extrinsic feedback can further be broken down into knowledge of results (KR) and knowledge of performance (KP). KR relates to the movement outcome in terms of the intended goal of the movement, while $\mathrm{KP}$ focuses on movement execution and relates to proper technique [6]. In previous research, variables related to KR have been examined such as timing, medium, frequency, and precision [7, 8] (for a review, see Schmidt [9] and Swinnen [10]). Regarding timing, feedback is generally better when presented with a brief delay after the movement is completed rather than immediately or concurrently [11]. Regarding the medium

Correspondence address: Kevin M. Fisher, Department of Physical Education and Sport, Central Michigan University, 177 Student Activity Center, Mount Pleasant, MI 48859, USA, e-mail: Fishe1k@cmich.edu

Received: September 14, 2019

Accepted for publication: October 21, 2020

Citation: Fisher KM, Kartes W, Gregorski M. Changing the type of knowledge of results affects the learning of a line-drawing task. Hum Mov. 2022;23(1):50-59; doi: https://doi.org/10.5114/hm.2021.104187. 
of feedback, KR has previously been defined as that which is provided in a verbal form, perceived via audition, and cognitively processed. This definition, with its emphasis on the potential verbal nature of KR, may have emerged as a result of factors related to sensory perception, communication, and information processing. First, practitioners and learners who can speak to one another via the same language are likely to express their thoughts or observations verbally owing to the natural inclination and efficiency with which verbal communication may occur. Secondly, there is a tendency of human beings to rely heavily on visual sensory information when perceiving their environment while potentially allowing it to supersede other senses (e.g., focusing too much on the optical outcome of a trial rather than the kinaesthetic awareness necessary to achieve habitual change in a movement pattern). Finally, there is a possibility that such intrinsic visual feedback could be redundant with KR, and thus practitioners strive to add new details or insight to what the learner may have just experienced. Because of these factors, other mediums of KR such as relevant visual or auditory information have not been examined in previous literature with the same level of detail as verbal KR. However, according to Sigrist et al. [12], technical advances in recent years have increased the possibilities to implement various types of unimodal feedback or multimodal augmented feedback. Regarding frequency of feedback, previous research by Winstein and Schmidt [8] has suggested that decreasing the frequency of KR over time enhances motor skill learning by reducing practice session performance but being beneficial to long-term retention and learning. Precision of feedback addresses the level of accuracy with which $\mathrm{KR}$ is presented, and this variable may change depending on whether qualitative or quantitative feedback is delivered. Qualitative feedback tends to subjectively describe a movement and informs the performer about areas such as level of correctness, perceived strengths and weaknesses, or surface features, such as force, speed, or distance [4]. Quantitative feedback tends to objectively describe a movement, is data driven, and indicates the direction and magnitude of error [13].

According to Kluger and DeNisi [14], hypotheses related to feedback interventions largely have their origins in the behaviouristic law of effect, which suggests that both positive (reinforcing) and negative (punishing) feedback interventions should facilitate performance and learning because the former increases the likelihood of a correct behaviour while the latter decreases the likelihood of an incorrect behaviour
$[15,16]$. Although much of Thorndike's research in learning theory involved animals, simple motor tasks were examined in humans as well (e.g., Bilodeau and Bilodeau [17], Dees and Grindley [18], MacPherson et al. [19, 20]). In a study by Trowbridge and Cason [21], the authors sought to further examine Thorndike's theory of learning using such a task that had been examined previously: line-drawing. Participants were blindfolded and asked to draw 3-inch lines on sheets of paper placed in front of them. Four testing conditions were utilized: blank (control), nonsense, rightwrong, and correct. In the blank testing condition, the participant was given no feedback. For the nonsense procedure, the experimenters spoke aloud a different nonsense syllable after each line-drawing attempt. In the right or wrong condition, the experimenter said 'right' if the participant was within $1 / 8$ of the 3 -inch line in either direction and 'wrong' in any other circumstance. In the correct procedure, experimenters also indicated that a line was right if it was within $1 / 8$ of 3 inches but provided the participant a specific error amount if it exceeded this threshold. The results in order from best to worst performance were as follows: correct, right-wrong, blank, and nonsense. The authors concluded that improvements in the task were due to the type and amount of information that was received by the participant, and the nonsense syllables acted to distract participants and produce performance decrements in comparison with the blank group. In a brief verbal survey that was administered to participants at the conclusion of the study, it was discovered that the principle cues utilized to perform the task were kinaesthetic cues from the arm and hand. However, several participants indicated that they visualized the length of the line that needed to be drawn and the distance of the required corrections.

The purpose of the current study was to further examine the conditions under which changing forms of KR might lead to improvements in a simple motor task. Specifically, researchers wanted to determine if factors such as increasing precision or utilizing a visual medium rather than a verbal one would foster learning. As a result of this goal, it is important to distinguish between motor performance and motor learning. Performance is measurable, observable behaviour that is susceptible to temporal variables such as motivation or fatigue. Learning is a more permanent neural process that results from practice and leads to the formation of motor memory and skill development. While these concepts are separate and distinct, they are closely connected because learning is inferred from performance [3]. As a result of these differences, the 
K. Fisher, W. Kartes, M. Gregorski, Changing knowledge of results in a line-drawing task

current study protocol utilized 3 phases: (a) acquisition, in which participants initially learned the task; (b) retention, in which participants were tested for learning outcomes after a 24-hour delay; and (c) transfer, in which participants were tested for skill generalizability after a 24-hour delay via a related but novel task. Understanding these phases is critical for motor learning research because both time and sequencing can influence learning effects in positive and negative directions [22, 23]. For the current study, KR was presented in 3 ways: (a) qualitatively vague, in which the line drawn was verbally reported to be correct, too long, or too short; (b) quantitatively precise, in which the line drawn was verbally reported to be correct or the exact error amount was given; and (c) visually precise, in which the error amount was shown to the participant by using a calliper as a visual aid. The hypotheses of this study were as follows:

1. In terms of performance and learning, the extrinsic KR groups will exceed the control group, who received no extrinsic feedback regarding performance.

2 . Increasing the precision of KR in the precise and visual groups will improve performance and learning when compared with the vague group.

3 . The medium of visual KR will be an effective alternative to precise verbal feedback as long as it presents information that is not redundant to the learner.

\section{Material and methods}

\section{Participants}

There were 48 participants, including 16 males and 32 females, aged $18-50$ years $(M=23.02, S D=6.04)$. This sample size was chosen by using a power estimate that was based on the previous line-drawing research by Trowbridge and Cason [21]. Mean error scores of $6.45,8.62,5.51$, and 1.62 were utilized along with standard deviation scores of 4.63, 4.65, 5.13, and 1.61. Power levels were set at 0.80 and alpha was set at 0.05 . On the basis of these results, it was determined that 48 participants would yield significant group differences. All participants were native English speakers, possessed the capability of drawing a straight line, and were naïve to the purpose of the study. The subjects were not placed under any time limit to perform the motor task. Day 1 of the study took approximately 1 hour while day 2 took approximately 20-30 minutes.

\section{Apparatus and task}

A fourth-generation iPad (Apple, Inc., Cupertino, USA) with the Drawing Desk application (4 Axis Solutions Ltd., Colombo, Sri Lanka) allowed participants to draw on the screen using the fingertip of the index finger of their preferred hand. The subjects were asked to close their eyes and draw what they perceived to be a 3-inch line over the course of 50 trials during practice. The following day, participants were asked to draw 20 three-inch lines to test task retention followed by 20 six-inch lines to test transfer. After each line was drawn, the application screen was cleared before beginning the next trial. Errors were measured to the nearest 0.001 of an inch; however, a bandwidth of acceptable error was utilized such that in all groups, the line was considered correct if it was within $1 / 8$ of an inch [24]. To assess error, a ruler was first used to establish 6 inches and the difference between this standard and the length of each line drawn was measured with an absolute origin digital calliper (iGaging OriginCal, San Clemente, USA), which extended just past 6 inches (6.154 inches) and measured to the nearest 0.001 of an inch. After each trial was complete, measurements were recorded in a spreadsheet on a laptop computer.

\section{Procedure}

This study occurred over 2 days, with the acquisition phase taking place on the first day and the testing phase taking place at approximately the same time on the second day. During acquisition, participants signed their informed consent, responded to a short verbal questionnaire regarding demographic information, and drew a 3-inch line for 50 trials. They were randomized into 4 different testing conditions, with each condition including 12 participants. The first condition was the control group (CTRL), provided with no extrinsic feedback after each drawing trial. According to Salmoni et al. [7], precision refers to manipulations that alter the accuracy of error information such as the number of figures presented or the smallest units of measurement (e.g., time, force, or distance). The least precise feedback offers simply qualitative terminology such as '(in)correct,' 'long,' 'short,' 'left,' or 'right'. Therefore, in the second condition, termed vague (VGE) owing to the nature of the precision, participants were only told if the line was correct, too short, or too long as a form of feedback. However, the precision of feedback can be increased by including both qualitative and quantitative elements. In conjunction with this 
greater precision, the third condition was termed precise (PRCS), and the individuals were informed if the line was correct or told the exact direction and magnitude of the error to the nearest 0.01 of an inch. In the final condition, termed visual (VSL), the subjects were informed if the line was correct or told the direction and shown the magnitude visually by displaying the gap in the calliper, without being able to see any numbers related to error. This fourth and final condition was so named because of the visual rather than verbal nature of the quantitative feedback. In all conditions, participants were asked to close their eyes while they drew the line and while they handed the tablet back to researcher, so they were unable to see the result of their movement and instead had to rely upon mental or kinaesthetic cues.

After a 24-hour delay, the subjects performed the testing phase, which first consisted of a retention test that was followed by a transfer test. During retention, participants were asked to draw a 3-inch line for 20 trials with no feedback. During transfer, they were given a more challenging task in which they were asked to draw a 6 -inch line for 20 trials without feedback. Accuracy values were recorded immediately after each trial. The initial phase (day 1) of this study was labelled as the acquisition phase while the second phase (day 2) was labelled as the testing or learning phase. The purpose of testing performance after a 24-hour delay was to separate the temporary effects of performance from the relatively permanent effects of retention.

\section{Dependent measures}

The dependent measure was the accuracy or inaccuracy of the length of each line produced. For example, if the line drawn was $1 / 4$ inch longer than the correct length, it was recorded as 0.25 , while a line that was drawn $1 / 4$ of an inch shorter than the correct length was recorded as -0.25 . If the line drawn was within $\pm 1 / 8$ of an inch (0.125), it was considered correct and recorded as an error amount of zero. Learning was assessed by examining the differences in the requested line length and the actual line length. Owing to the generally positive result of performance repetitions on a motor skill, it was not expected that there would be any negative effects on learning.

\section{Data treatment and analyses}

The acquisition phase, which consisted of 50 trials, was split into 10 blocks, each consisting of 5 trials.
To assess differences in performance between groups during acquisition, a 4 (group) $\times 10$ (block) repeated-measures ANOVA was performed. To assess differences during retention and transfer, a 4 (group) $\times 2$ (testing condition) repeated-measures ANOVA was performed. Accuracy was examined with measures of constant error (CE) and absolute CE (ACE), while consistency was determined with variable error (VE). $\mathrm{CE}$ measured the response bias in relation to a target measure, ACE measured the magnitude of the error without considering direction, and VE measured the consistency of the movement. For all post-hoc pairwise comparisons, Sidak analyses were performed. For violations of sphericity, Greenhouse-Geisser corrections were used. The alpha level for all analyses was set at 0.05 and partial eta squared values were noted and subsequently reported where appropriate. Variables for the study were normally distributed.

\section{Ethical approval}

The research related to human use has complied with all the relevant national regulations and institutional policies, has followed the tenets of the Declaration of Helsinki, and has been approved by the institutional Internal Review Board.

\section{Informed consent}

Informed consent has been obtained from all individuals included in this study.

\section{Results}

Acquisition (practice)

\section{Absolute constant error ${ }^{1}$}

A repeated-measures ANOVA revealed a nonsignificant block $\times$ condition interaction: $F(9.67,44)=$ $0.647, p=0.766, \eta^{2}=0.042$. There was a main effect for block: $F(3.22,44)=6.07, p<0.001, \eta^{2}=0.121$, indicating improved accuracy with practice. Follow-up Sidak comparisons indicated that all feedback groups outperformed the CTRL group $(p<0.05)$ (Figure 1). For ACE, the CTRL group averaged $0.449(S D=0.095)$, the VGE group averaged $0.198(S D=0.075)$, the PRCS group averaged $0.132(S D=0.067)$, and the VSL group averaged $0.154(S D=0.079)$. These degrees of free-

\footnotetext{
${ }^{1}$ Constant error was also examined for each of the study phases but yielded nonsignificant results, presumably owing to the cancelling of directionally-opposite scores. Since constant error yielded no additional insight into performance patterns, it is omitted here.
} 


\section{HUMAN MOVEMENT}

K. Fisher, W. Kartes, M. Gregorski, Changing knowledge of results in a line-drawing task
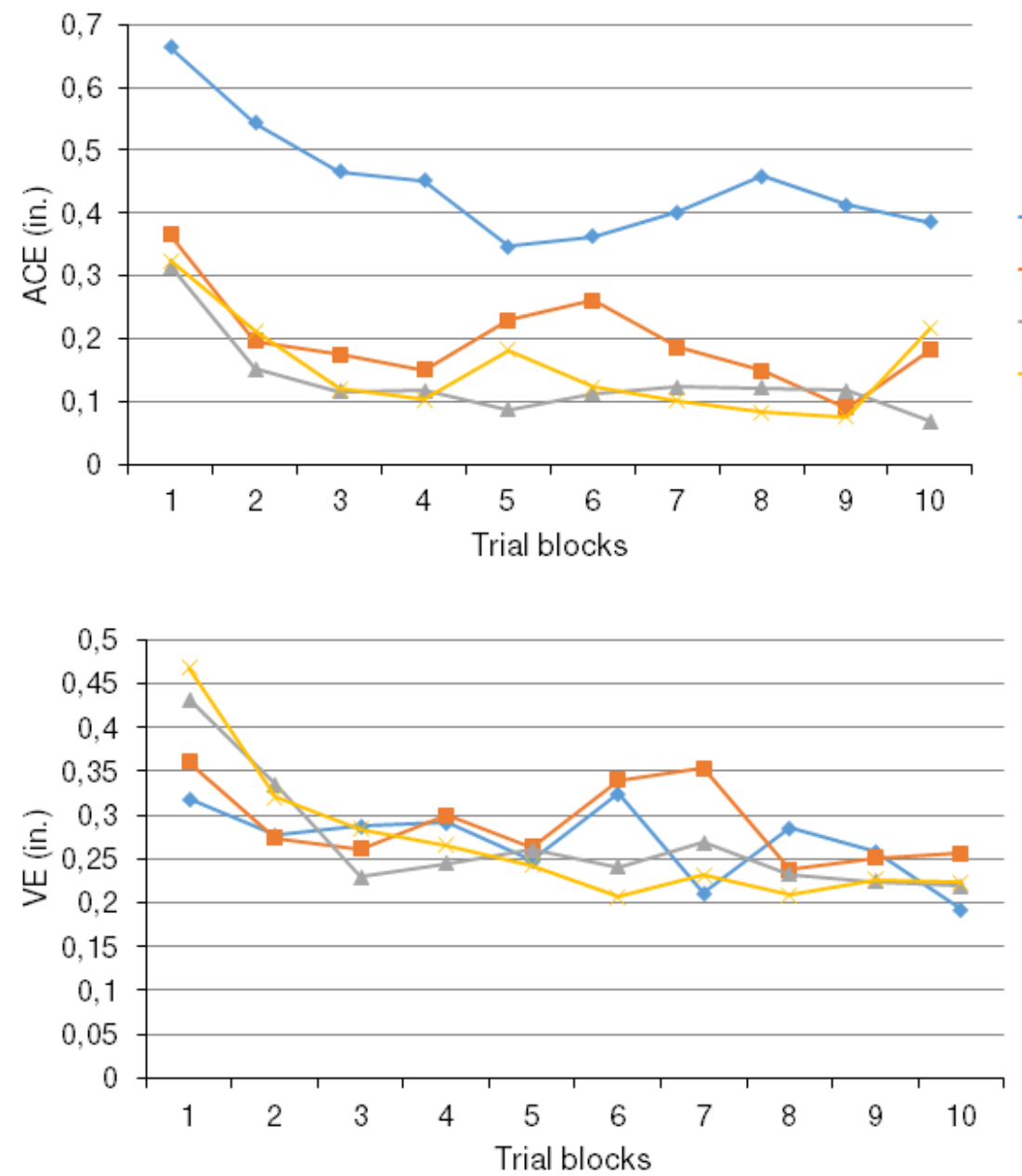

CTRL - control group

VGE - vague feedback group PRCS - precise feedback group

VSL - visual feedback group

Figure 1. Absolute constant error (ACE) during acquisition for all conditions

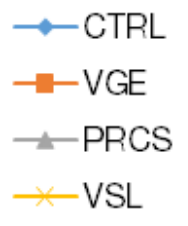

Figure 2. Variable error (VE) during acquisition for all conditions dom were the result of using Greenhouse-Geisser corrections where sphericity was violated.

\section{Variable error}

A repeated-measures ANOVA revealed a nonsignificant block $\times$ condition interaction: $F(15.76,44)=$ 1.22, $p=0.258, \eta^{2}=0.076$. There was a main effect for block: $F(5.26,44)=6.36, p<0.001, \eta^{2}=0.126$, indicating improved consistency with practice. Followup Sidak comparisons indicated no significant differences between groups (Figure 2). For VE, the CTRL group averaged $0.269(S D=0.043)$, the VGE group averaged $0.290(S D=0.046)$, the PRCS group averaged $0.269(S D=0.067)$, and the VSL group averaged $0.269(S D=0.079)$.

\section{Retention and transfer (testing)}

\section{Absolute constant error}

A repeated-measures ANOVA revealed a nonsignificant test $\times$ condition interaction: $F(3,44)=1.99$, $p=0.130, \eta^{2}=0.119$. There was a main effect for test: $F(1,44)=37.56, p<0.001, \eta^{2}=0.461$ (Figure 3). For retention testing, follow-up Sidak comparisons indicated no significant differences (Figure 4); however, for transfer testing, follow-up Sidak comparisons indicated that the CTRL group was significantly outperformed by the VSL group (Figure 5).

In retention testing (Figure 4), the CTRL group averaged $0.328(S D=0.365)$, the VGE group averaged $0.284(S D=0.260)$, the PRCS group averaged 0.184 $(S D=0.147)$, and the VSL group averaged $0.156(S D=$ 0.136). In transfer testing (Figure 5), the CTRL group averaged $1.09(S D=0.640)$, the VGE group averaged $0.564(S D=0.524)$, the PRCS group averaged 0.651 $(S D=0.469)$, and the VSL group averaged $0.502(S D=$ $0.360)$.

\section{Variable error}

A repeated-measures ANOVA revealed a nonsignificant test $\times$ condition interaction: $F(15.76,44)=$ 1.22, $p=0.258, \eta^{2}=0.076$. There was a main effect for test: $F(9,44)=6.36, p<0.001, \eta^{2}=0.126$, such that 
K. Fisher, W. Kartes, M. Gregorski, Changing knowledge of results in a line-drawing task

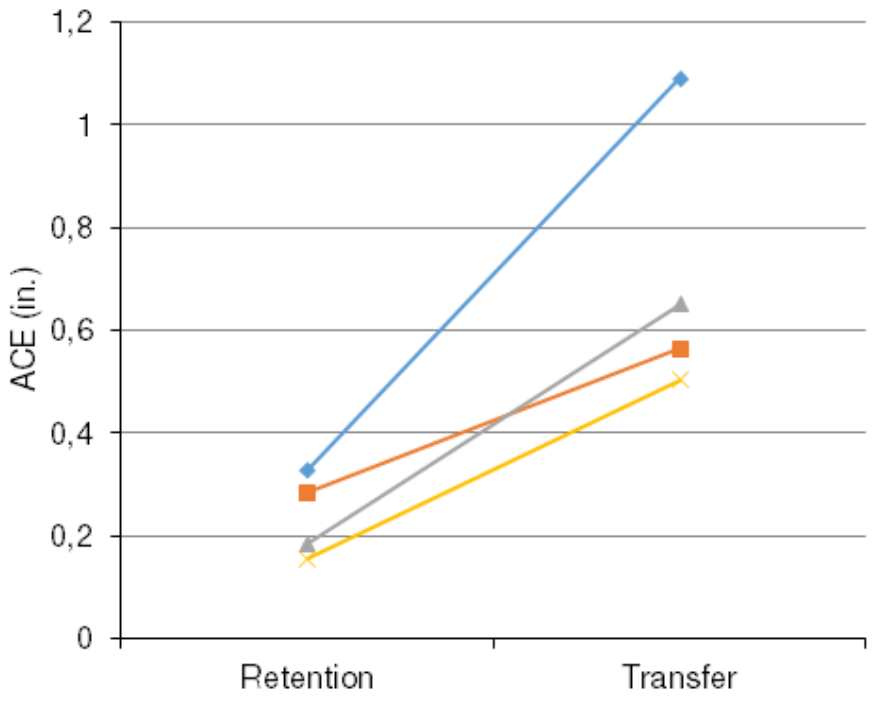

CTRL - control group

VGE - vague feedback group

PRCS - precise feedback group

VSL - visual feedback group

Figure 3. Comparison of absolute constant error (ACE) across retention and transfer

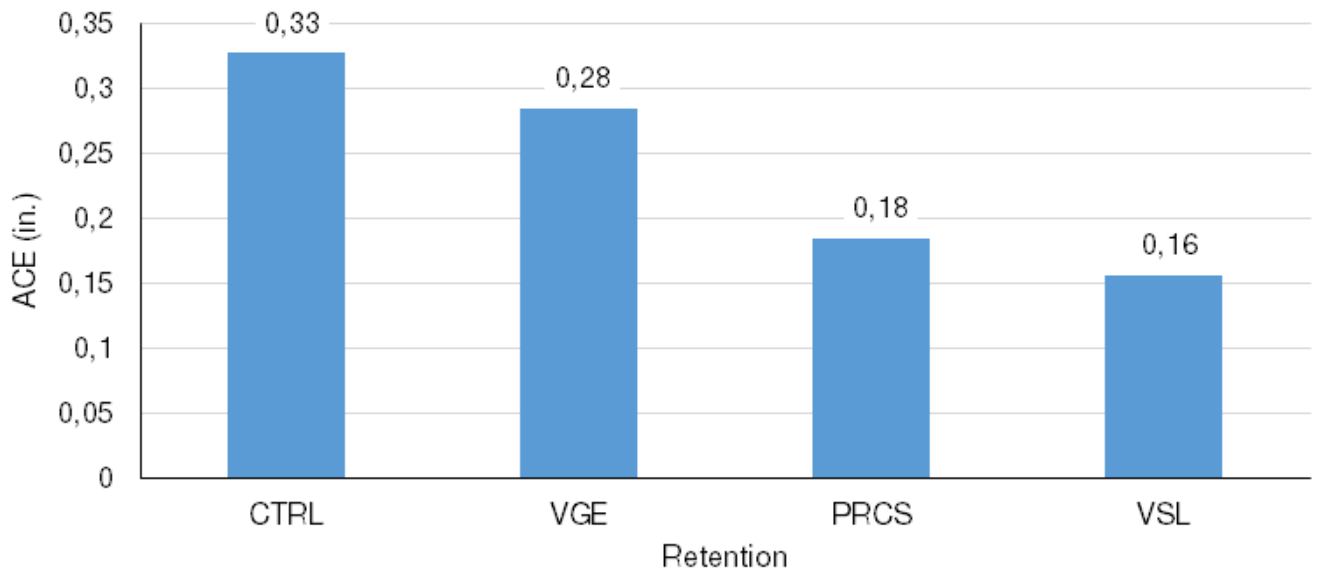

CTRL - control group

VGE - vague feedback group PRCS - precise feedback group VSL - visual feedback group

Figure 4. Comparison of absolute constant error (ACE) for all groups during retention testing

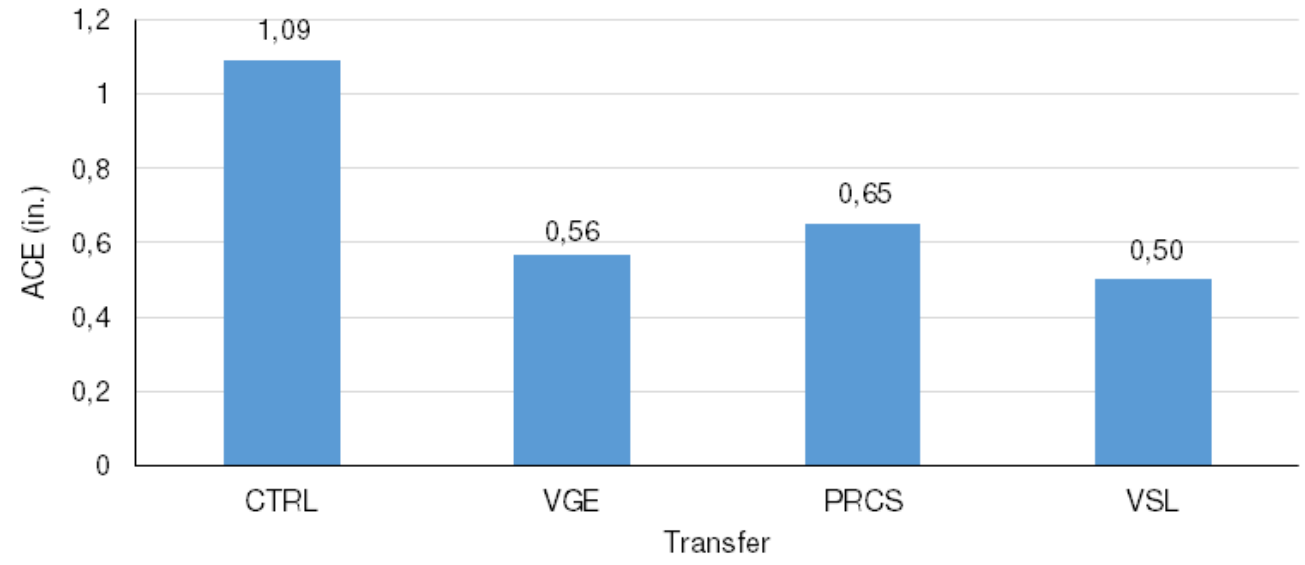

CTRL - control group

VGE - vague feedback group PRCS - precise feedback group VSL - visual feedback group

Figure 5. Comparison of absolute constant error (ACE) for all groups during transfer testing 
K. Fisher, W. Kartes, M. Gregorski, Changing knowledge of results in a line-drawing task

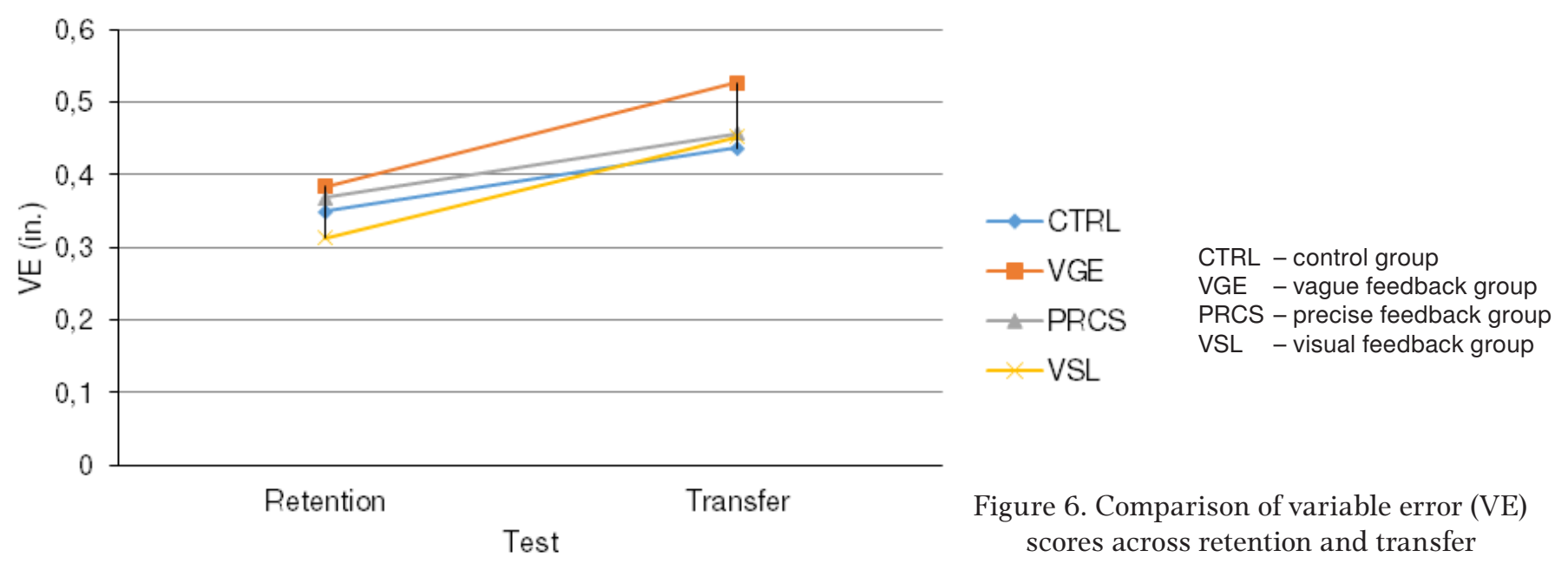

participants demonstrated more consistency in retention than transfer (Figure 6). Follow-up Sidak comparisons indicated no significant differences between groups.

In retention testing, the CTRL group averaged 0.349 $(S D=0.195)$, the VGE group averaged $0.384(S D=$ $0.185)$, the PRCS group averaged $0.368(S D=0.117)$, and the VSL group averaged $0.312(S D=0.09)$. In transfer testing, the CTRL group averaged $0.437(S D=$ $0.124)$, the VGE group averaged $0.526(S D=0.177)$, the PRCS group averaged $0.457(S D=0.109)$, and the VSL group averaged $0.451(S D=0.107)$.

\section{Discussion}

The purpose of this study was to examine the conditions under which changing forms of KR might lead to improvements in a simple motor task by determining if factors such as increasing precision of KR or utilizing a visual medium rather than a verbal one would foster learning. In comparison with a control condition that received no extrinsic feedback, 3 types of KR were examined: qualitative vague KR, quantitative precise $K R$, and visually precise KR. It was hypothesized that participants in the 3 extrinsic feedback groups would exceed the CTRL group in terms of performance and learning. This hypothesis was partially supported; participants in the VGE, PRCS, and VSL groups outperformed those in the CTRL group during acquisition, suggesting that extrinsic feedback and, specifically, qualitative or quantitative KR, can enhance performance on a line-drawing task. Significant differences between groups were not observed during retention testing, but data indicated trends in this direction (Figure 4). Finally, significant differences between the CTRL and VSL groups were observed in transfer testing, and both the VGE and PRCS groups

demonstrated lower error scores than the CTRL group, adding support to this hypothesis (Figure 5). Upon an examination of acquisition scores, results seem to support those obtained by Thorndike [16], Trowbridge and Cason [21], or Bilodeau et al. [25], who suggest that learning requires both sufficient practice and an understanding of stimulus-response pairings (i.e., connections between movements and outcomes). Proponents of such a behaviouristic learning theory may advocate that error scores cannot be significantly reduced in the absence of feedback, and thus KR is a necessary component to the improvement and learning of even a simple motor task such as line-drawing. However, results from retention and transfer testing were mixed. Retention testing showed no significant differences between groups, implying that the CTRL group, while seeming to struggle during acquisition, was learning components of the task that would aid performance later. These testing results support the idea that KR in a simple motor task such as line-drawing may be beneficial to learning but is not a requisite component of learning. When examining changes in experimental performance over the course of multiple days, it may also be pertinent to consider psychological factors such as familiarity with the task or procedure, comfort level in the experimental setting, or the influences of emotions such as confidence, boredom, or frustration. It may be the case that participants in all groups felt comfortable with the task and protocol after the acquisition phase, which aided performance during testing. However, groups that received feedback may have experienced higher levels of frustration with the task since their success or failure was reported after each trial.

The second hypothesis of this study stated that increasing the precision of KR would improve performance and learning. This hypothesis was not sup- 
ported; participants in the VGE group performed similarly to the PRCS and VSL groups, although the PRCS and VSL groups experienced higher levels of feedback precision and should have been able to apply this additional information to correct their errors more accurately [7]. However, according to Anderson et al. [5], more precision may not necessarily be better in terms of extrinsic feedback. If the detail of the precision is too great or if less feedback is sufficient to successfully complete the task, the additional information is likely to be discarded. These similarities in performance between the groups may be due to limitations of the current study, including task complexity and the units of measurement that were utilized. The task examined was relatively simplistic in terms of cognitive and motor demands. A task of greater complexity (i.e., more limbs involved, steps, or dimensions of error) may have been more apt to tease apart performance differences between the various KR groups because of the increased possibility for errors and the value of feedback in correcting them. Additionally, the units used in this study were standard inches, which all participants had likely been familiar with previously, even if to varying degrees. In retrospect, it may have been more equitable and demanding to use a new, random unit of measurement that participants would have to learn and adjust to as they gained experience in the study (i.e., an imaginary unit). This methodological change may have provided a better opportunity for the subjects in separate groups to demonstrate greater improvements and learning effects.

Finally, the third hypothesis of this study stated that precise visual KR would be an effective alternative to precise verbal KR. This hypothesis was supported; there were no significant differences in performance or learning between the VSL and PRCS groups, suggesting that novel, augmented visual KR related to the performance of a simple motor skill could effectively convey movement error and lead to improvement. During transfer testing, differences in performance were found to be greater between the VSL and CTRL groups than the PRCS and CTRL groups, suggesting that, where appropriate, visual KR could act as an equally potent but more efficient means of communicating information. Consider, for example, a coach who could show an athlete a snapshot of their outcome rather than explaining the results in detail. Assuming that the necessary technology is available, such a scenario could be beneficial to both parties in terms of the accuracy, consistency, and efficiency of information dissemination. These results suggest that the verbal aspect of KR not be emphasized when defining KR as in Sal- moni et al. [7]. In instances such as the current study where a visual aid may be used to provide $\mathrm{KR}$ or in a musical or rhythmical setting where an auditory aid may be used, it seems evident that the medium can change with the context and can be equally effective as verbal KR, provided that the information presented is not redundant with intrinsic feedback.

These findings support those of previous researchers who have concluded that augmented feedback is an important determinant of motor learning, and variables regarding KR such as precision and medium are critical to consider (e.g., Liu and Wrisberg [26], Sigrist et al. [12]). These results also support and extend those achieved by Trowbridge and Cason [21], who determined that improvements in a line-drawing task were due to the type and amount of information provided. Specifically, these observations indicate that performance may improve through verbal or visual feedback and that the latter medium may be an effective alternative to feedback that is presented verbally and perceived via audition. Movement practitioners such as physical educators, coaches, or therapists who have limited time with learners (e.g., students, athletes, or clients) are likely striving to find the most effective means of communicating feedback to motor skill learners in a timely fashion. These findings suggest that $\mathrm{KR}$ in a visual medium may be as effective as a verbal explanation and more efficient in terms of delivery and communication. Additionally, it is possible that participants who readily utilize mental imagery or visual cues may benefit more from visual feedback, although more research is needed in this area.

\section{Limitations}

Broadly, this study addressed the unique demands of performing a simple motor task that involved drawing a digital line of a required length. Specifically, the structure of this project consisted of drawing a line on a tablet, performed in a controlled environment. This type of motor task may not generalize to environments outside of the purview of this study [27]. Participants for this task ranged in age from 18 to 50 years, which constitutes a gap of 32 years. With such a large range, there is a possibility that age-related variables such as dexterity, fine motor control, or visual acuity could play a role in the results. Additionally, as mentioned previously, a more complex motor task that featured a novel error metric in which participants had to gain understanding and familiarity may have been better able to discern group differences depending on types or degrees of feedback. 
K. Fisher, W. Kartes, M. Gregorski, Changing knowledge of results in a line-drawing task

\section{Conclusions and future directions}

In conclusion, the results of this study (1) support previous research that indicates that feedback in the form of both qualitative and quantitative KR that is timely, relevant, and precise can facilitate performance and learning of a motor skill in comparison with conditions in which no feedback is present. This finding was evidenced by the improved performance of the 3 feedback groups in comparison with the control group. These results also (2) extend previous research by suggesting that visual forms of KR, when available, may constitute an effective alternative to lengthier verbal explanations of error. This finding was evidenced by the similar improvements in performance observed in the group that received precise verbal KR and the group that received visual KR. Such a visual mechanism may be helpful to individuals who regularly utilize imagery as a facilitator of learning.

Future directions of research should include testing specific populations that movement practitioners like educators or coaches often interact with such as children or athletes. Indeed, previous research suggests that there may be differences between children and adults in learning and performance of tasks involving fine motor skills and manual dexterity [28]. Future studies could also utilize more complex motor tasks to generalize potential benefits of increasing KR precision or applying visual forms of feedback. Finally, other mediums of feedback such as auditory clips, kinaesthetic cues, or video replay could be examined for tasks in which these are practical or appropriate.

\section{Disclosure statement}

No author has any financial interest or received any financial benefit from this research.

\section{Conflict of interest}

The authors state no conflict of interest.

\section{References}

1. Sharma DA, Chevidikunnan MF, Khan FR, Gaowgzeh RA. Effectiveness of knowledge of results and knowledge of performance in the learning of a skilled motor activity by healthy young adults. J Phys Ther Sci. 2016; 28(5):1482-1486; doi: 10.1589/jpts.28.1482.

2. Magill R, Anderson D. Motor learning and control: concepts and applications, $11^{\text {th }}$ ed. New York: McGrawHill Education; 2017.

3. Schmidt RA, Lee TD. Motor learning and performance: from principles to applications, $6^{\text {th }}$ ed. Champaign: Human Kinetics; 2019.
4. Weeks DL, Kordus RN. Relative frequency of knowledge of performance and motor skill learning. Res Q Exerc Sport. 1998;69(3):224-230; doi: 10.1080/0270 1367.1998.10607689.

5. Anderson DI, Magill RA, Mayo AM, Steel KA. Enhancing motor skill acquisition with augmented feedback. In: Hodges NJ, Williams AM (eds.), Skill acquisition in sport: research, theory and practice, $3^{\text {rd }}$ ed. Abingdon: Routledge; 2020; 3-19.

6. Schmidt RA, Lee TD, Winstein CJ, Wulf G, Zelaznik HN. Motor control and learning: a behavioral emphasis, $6^{\text {th }}$ ed. Champaign: Human Kinetics; 2018.

7. Salmoni AW, Schmidt RA, Walter CB. Knowledge of results and motor learning: a review and critical reappraisal. Psychol Bull. 1984;95(3):355-386; doi: 10.1037/ 0033-2909.95.3.355.

8. Winstein CJ, Schmidt RA. Reduced frequency of knowledge of results enhances motor skill learning. J Exp Psychol Learn Mem Cogn. 1990;16(4):677-691; doi: 10.1037/0278-7393.16.4.677.

9. Schmidt RA. Frequent augmented feedback can degrade learning: evidence and interpretations. In: Requin J, Stelmach GE (eds.), Tutorials in motor neuroscience. Dordrecht: Kluwer Academic Publishers; 1991; 59-75.

10. Swinnen SP. Information feedback for motor skill learning: a review. In: Zelaznik HN (ed.), Advances in motor learning and control. Champaign: Human Kinetics; 1996; 37-66.

11. Swinnen SP, Schmidt RA, Nicholson DE, Shapiro DC. Information feedback for skill acquisition: instantaneous knowledge of results degrades learning. J Exp Psychol Learn Mem Cogn. 1990;16(4):706-716; doi: 10.1037/0278-7393.16.4.706.

12. Sigrist R, Rauter G, Riener R, Wolf P. Augmented visual, auditory, haptic, and multimodal feedback in motor learning: a review. Psychon Bull Rev. 2013;20(1):2153; doi: 10.3758/s13423-012-0333-8.

13. Niznikowski T, Sadowski J, Mastalerz A. The effectiveness of different types of verbal feedback on learning complex movement tasks. Hum Mov. 2013;14(2):148153; doi: 10.2478/humo-2013-0009.

14. Kluger AN, DeNisi A. The effects of feedback interventions on performance: a historical review, a meta-analysis, and a preliminary feedback intervention theory. Psychol Bull. 1996;119(2):254-284; doi: 10.1037/00 33-2909.119.2.254.

15. Thorndike EL. Educational psychology. Vol. 1: The original nature of man. New York: Columbia University, Teachers College; 1913.

16. Thorndike EL. The law of effect. Am J Psychol. 1927; 39(1/4):212-222; doi: 10.2307/1415413.

17. Bilodeau EA, Bilodeau IM. Variable frequency of knowledge of results and the learning of a simple skill. J Exp Psychol. 1958;55(4):379-383; doi: 10.1037/h0043214.

18. Dees V, Grindley GC. The effect of knowledge of results on learning and performance. IV: The direction of the 
error in very simple skills. Q J Exp Psychol. 1951;3(1): 36-42; doi: 10.1080/17470215108416770.

19. MacPherson SJ, Dees V, Grindley GC. The effect of knowledge of results on learning and performance. II: Some characteristics of very simple skills. Q J Exp Psychol. 1948;1(2):68-78; doi: 10.1080/17470214808 416747.

20. MacPherson SJ, Dees V, Grindlgey GC. The effect of knowledge of results on learning and performance. III: The influence of the time interval between trials. Q J Exp Psychol. 1949;1(4):167-174; doi: 10.1080/1747021 4908416762.

21. Trowbridge MH, Cason H. An experimental study of Thorndike's theory of learning. J Gen Psychol. 1932; 7(2):245-260; doi: 10.1080/00221309.1932.9918465.

22. Lee TD. Transfer-appropriate processing: a framework for conceptualizing practice effects in motor learning. Adv Psychol. 1988;50:201-215; doi: 10.1016/S01664115(08)62557-1.

23. Shea JB, Morgan RL. Contextual interference effects on the acquisition, retention, and transfer of a motor skill. J Exp Psychol Hum Learn Mem. 1979;5(2):179187; doi: 10.1037/0278-7393.5.2.179.

24. Sherwood DE. Effect of bandwidth knowledge of results on movement consistency. Percept Mot Skills. 1988;66(2):535-542; doi: 10.2466/pms.1988.66.2.535.

25. Bilodeau EA, Bilodeau IM, Schumsky DA. Some effects of introducing and withdrawing knowledge of results early and late in practice. J Exp Psychol. 1959;58(2): 142-144; doi: 10.1037/h0040262.

26. Liu J, Wrisberg CA. The effect of knowledge of results delay and the subjective estimation of movement form on the acquisition and retention of a motor skill. Res $\mathrm{Q}$ Exerc Sport. 1997;68(2):145-151; doi: 10.1080/0270 1367.1997.10607990.

27. Wulf G, Shea CH. Principles derived from the study of simple skills do not generalize to complex skill learning. Psychon Bull Rev. 2002;9(2):185-211; doi: 10.37 58/bf03196276.

28. Czajka K, Kołodziej M, Kochan K, Sławińska T. Development of manual dexterity in preschool children. Hum Mov. 2018;19(4):79-86; doi: 10.5114/hm.2018. 79735. 